\title{
CONVOLUTION AND DIFFERENTIAL SUBORDINATION
}

\author{
T.N. SHANMUGAM \\ Department of Mathematics \\ Anna University \\ Madras 600 025, India \\ (Received July 15, 1987)
}

ABSTRACT. Let A denote the class of all functions $f$ analytic in the open unit disc $U$ with $f(0)=0=f^{\prime}(0)-1$. Let $h$ be any convex univalent analytic function on $U$ with $h(0)=1$ and $\operatorname{Re} h(z)>0$ in $U$. Let $g E A$ be fixed. Denote by $s_{g}(h)$ the class of all functions $f \varepsilon A$ such that, $g^{\star} f(z) \neq 0$ in $U$ and $\frac{a\left(g g^{\star}\right)^{\prime}(z)}{\left(g^{\star} f\right)(z)}<h(z), z \varepsilon U(<\operatorname{denote}$ subordination). It is proved in this paper that the class $s_{g}(h)$ is closed under convolution with convex functions. It has also been established that $S_{g}(h) \subseteq$ $S_{\Phi^{*} \mathrm{~g}}(\mathrm{~h})$ where $\Phi$ is any convex univalent function in $A$. Four other classes are also defined and studied using mainly the convex hull method and the methd of differential subordination.

KEY WORDS AND PHRASES. Convolution, Convex function, Close-to-convex function, Quasiconvex function, Starlike function, Subordination.

1980 AMS SUBJECT CLASSIFICATION CODE. Primary 30C45.

1. INTRODUCTION.

Let $U=\{z:|z|<1\}$ and $H(U)$ be the class of all holomorphic functions defined on $U$. Let $A=\left\{f \notin H(U) / f(0)=0, f^{\prime}(0)=1\right\}$. Let $f, g \varepsilon H(U)$ and $f(z)=\sum_{0}^{\infty} b_{n^{2}} z^{n}$.

Then the convolution of $f$ and $g$ is denoted as

$$
(f * g)(z)=\sum_{0}^{\infty} a_{n} b_{n} z^{n} .
$$

Let $g$ and $G \mathrm{eH}(U), g(z)$ is said to be subordinate to $G(z)$ (written $g(z)<G(Z)$ ) in $U$ if $G(z)$ is univalent in $U, g(0)=G(0)$ and $g(U) \subseteq G(U)$. Let $S^{\star}, K, Q$ and $C$ denote the subclass of A consisting of Starlike univalent, convex univalent, Quasi-Convex and close-to-convex functions respectively. Let $M_{\alpha}$ denote the class of functions in $A$ which are $\alpha$-convex (Mocanu sense) in $\mathrm{U}$. 
As a general reference for the definitions and properties of the above classes the reader may consult the book by A.W. Goodman [1].

The aim of this paper is to give various subclasses of $A$, analogous to the classes $S *, K, Q, C$ and $M_{\alpha}$, and to study some properties of the new classes.

2. BASIC THEOREMS AND DEFINITLON OF THE NEW CLASSES.

We need the following theorems to prove our main results.

THEOREM 2.1 [2]: Let $\phi \varepsilon A$ be convex univalent, $g \varepsilon S^{*}$ and $F \varepsilon H(U)$ such that $\operatorname{ReF}(z)$

$>0$ for $\forall z \varepsilon U$. Then $\frac{\phi^{*} F g}{\phi^{*} g}$ lies in the convex hull of $F(U)$.

THEOREM 2.2 [3]: Let $B, \nu \in C, h \in H(U)$ be convex univalent in $U$ with $h(0)=1$ and $\operatorname{Re}(\beta h(z)+\nu)>0, \forall z \varepsilon U$ and let

$$
\begin{aligned}
& p(z)=1+p_{1} z+\ldots \varepsilon H(U) \text {. Then } \\
& p(z)+\frac{z p^{\prime}(z)}{B p(z)+\nu}<h(z) \Rightarrow p(z)<h(z)
\end{aligned}
$$

A modification of Theorem 2.2 is given in

THEOREM 2.3 [4]: Let $\beta, \nu \varepsilon C, h \varepsilon H(U)$ be convex univalent in $U$ with $h(0)=1$ and $\operatorname{Re}(\beta h(z)+\nu)>0, \forall z \varepsilon U$ and let $q \varepsilon H(U)$ with $q(0)=1$ and $q(z)<h(z), \quad \forall z \varepsilon U$. If

$$
\begin{aligned}
& p(z)=1+p_{1} z+\ldots \quad \text { is in } H(U) \text {, then } \\
& p(z)+\frac{z p^{\prime}(z)}{B q(z)+\nu}<h(z) \Rightarrow p(z)<h(z) .
\end{aligned}
$$

To avoid repetition we say once and for all in this paper, unless otherwise specified, $g$ will denote a fixed function in $A$ and $h$ will always denote a convex univalent function on $U$ with $h(0)=1$ and $\operatorname{Re} h(z)>0$ for $\forall z \varepsilon U$.

DEFINITION 2.1: Let $S_{g}(h)$ denote the class of all functions $f \varepsilon A$ such that $\frac{(g * f)(z)}{z} \neq 0$ in $U$ and satisfying

$$
\frac{z(g * f)^{\prime}(z)}{(g * f)(z)}<h(z)
$$

DEFINITION 2.2: Let $\mathrm{K}_{g}(h)$ denote the class of all functions $f \varepsilon A$ such that $(g * f)^{\prime}(z) \neq 0$ in $U$ and satisfying

$$
1+\frac{z(g * f)^{\prime \prime}(z)}{(g * f)^{\prime}(z)}<h(z)
$$

DEFINITION 2.3: Denote by $C_{g}(h)$ the class of all functions $f \varepsilon A$ such that $\frac{(g \star f)}{z}(z) \neq 0$ for $\forall z \in U$ and satisfying

$$
\frac{z(g * f)^{\prime}(z)}{(g * \Psi)(z)}<h(z), \forall z \varepsilon U, \text { for some } \Psi \varepsilon S_{g}(h)
$$


REMARK 2.1: If $g(z)=z /(1-z)^{a}$ (a real) then $S_{g}(h)$ and $C_{g}(h)$ coincides with the classes $S_{a}(h)$ and $C_{a}(h)$ respectively introduced in [4]. $K_{g}(h)$ is the class $K_{a}(h)$ introduced in [5]. For the choice $h(z)=\frac{1+z}{1-z}$, the classes $S_{a}(h)$ and $K_{a}(h)$ were investigated by $S$. Owa et al [6]. If $g(z)+\frac{z}{(1-z)} \frac{z}{2(1-\alpha)}$ and $h(z)=\frac{1+(1-2 \alpha) z}{1-z}$, $0<\alpha<1$, then $\mathrm{s}_{\mathrm{g}}(\mathrm{h})$ is nothing but the class of pre-starlike functions of order $\alpha$ introduced by Ruschewegh in [7].

If $g(z)$ is taken as $z$ then $S_{g}(h)$ is the class $A$ and hence in general functions in $\mathrm{S}_{\mathrm{g}}(\mathrm{h})$ need not be univalent.

DEFINITION 2.4: Let $\alpha$ be any real number. Let $\mathrm{K}_{g}^{\alpha}(\mathrm{h})$ denote the class of functions $f \in A$ such that $\frac{\left(g \star_{f}\right)(z)}{z} \neq 0$ and $\left(g \star_{f}\right)^{\prime}(z) \neq 0$ in $U$ and

$$
J_{g}(\alpha ; f(z))=\alpha\left(1+\frac{z(g * f)^{\prime \prime}(z)}{(g \star f)^{\prime}(z)}\right)+(1-\alpha) \frac{z(g * f)^{\prime}(z)}{(g * f)(z)}
$$

is subordinate to $h(z)$.

REMARK 2.2: When $g(z)=z /(1-z)^{a} k_{g}^{\alpha}(h)$ is the same as $k_{a}^{\alpha}(h)$ introduced in [5]. As it can be seen clearly that $K_{g}^{o}(h)=s_{g}(h)$ and $K_{g}^{\prime}(h)=K_{g}(h), K_{g}^{\alpha}(h)$ provides a 'continuous passage' from the class $K_{g}(h)$ to $S_{g}(h)$ as a decreases from 1 to 0.

DEFINITION 2.5: Let $Q_{g}(h)$ denote the class of all functions $f \varepsilon A$ such that $\frac{\left(g \star_{f}\right)(z)}{z} \neq 0$ in $U$ and satisfying for some $\Phi \varepsilon K_{g}(h)$

$$
\frac{\left[z(g * f)^{\prime}(z)\right]^{\prime}}{(g * \Phi)^{\prime}(z)}<h(z), \quad \forall z \varepsilon U
$$

REMARK 2.3: When $g(z)=z /(1-z)^{a}$ we shall denote the class $Q_{g}(h)$ by $Q_{a}(h)$. If further $a=1$ and $h(z)=\frac{1+z}{1-z}, Q_{a}(h)$ is the class of Quasi-Convex functions introduced by K.I. Noor and D.K. Thomas [8].

\section{MAIN THEOREMS.}

THEOREM 3.1: If $f \varepsilon S^{*}(h)$ and $g$ is a convex function then $f \varepsilon S(h)$.

PROOF: We have

$$
\frac{z(g * f)^{\prime}(z)}{(g * f)(z)}=\frac{\left(g * z f^{\prime}\right)(z)}{(g \star f)(z)}=\frac{\left(g * \frac{z f^{\prime}}{f} \cdot f\right)(z)}{(g * f)(z)}
$$

Since $f \varepsilon S^{*}(h), \operatorname{Re}\left(\frac{z f^{\prime}(z)}{f(z)}\right)>0$ and $g$ convex, and hence by an application of Theorela $A$ we get,

$$
\frac{z(g * f)^{\prime}(z)}{(g * f)(z)}<h(z)
$$

which implies $f \varepsilon \mathrm{S}_{\mathrm{g}}(\mathrm{h})$. 
It is a well known fact that $K \subseteq S^{*}$ and $f \varepsilon K$ if and only $z f^{\prime} \varepsilon S^{*}$, we shall now extend this fact to the class $S_{g}(h)$ and $K_{g}(h)$.

THEOREM 3.2: (i) $\mathrm{K}_{\mathrm{g}}(\mathrm{h}) \subseteq \mathrm{S}_{\mathrm{g}}(\mathrm{h})$

(ii) $\quad f \in K_{g}(h)$ if and on $1 y$ if $z f^{\prime} \varepsilon S_{g}(h)$

PROOF: (i) Let $p(z)=\frac{z\left(g^{\star} f\right)^{\prime}(z)}{\left(g^{\star} f\right)(z)}$, Logarithamic derivative of $p(z)$ and a nultiplication by $z$ gives

$$
p(z)+\frac{z p^{\prime}(z)}{p(z)}=1+\frac{z\left(g *_{f}\right)^{\prime \prime}(z)}{(g * f)(z)}
$$

If $f \in K_{g}(h)$ then by Definition 2.2 the right side of (3.1), and hence the left side is subordinate, to $h(z)$. Applying Theorem 2.2 we get, $p(z)<h(z)$ and hence the result (i). To prove (ii) we have for any two functions $f$, geA such that $\frac{(f * g)(z)}{z} \neq 0,(f * g)^{\prime}(z) \neq 0$.

$$
\frac{z\left(g * z f^{\prime}\right)^{\prime}(z)}{\left(g * z f^{\prime}\right) \star(z)}=1+\frac{z(g * f)^{\prime} \cdot(z)}{(g * f)^{\prime}(z)} .
$$

Now, if $f \in K_{g}(h)$ then from $(2.2)$ and $(3.2) \frac{z\left(g^{\star} z f^{\prime}\right)^{\prime \prime}(z)}{\left(g^{\star} z f^{\prime}\right)(z)}<h(z)$. Therefore $z f^{\prime} \varepsilon S_{g}(h)$. Conversly, if $z f^{\prime} \varepsilon S_{g}(h)$, from $(2.1)$ and $(3.2)$,

$1+\frac{z(g * f)^{\prime} \cdot(z)}{(g * f)^{\prime}(z)}<h(z)$ and hence $f \varepsilon K_{g}(h)$

REMARK 3.1: For $g(z)=z /(1-z)^{a}$ Theorem 3.1 gives Theorem 3 in [5] as a particular case.

Next we prove the classes $\mathrm{S}_{\mathrm{g}}(\mathrm{h})$ and $\mathrm{K}_{\mathrm{g}}(\mathrm{h})$ are closed under convolutions with convex univalent functions.

THEOREM 3.3: Let $\Phi \varepsilon_{A}$ be convex univalent then for every $f \in S_{g}(h), \Phi \star_{f} \varepsilon S_{g}(h)$.

PROOF: Let $F(z)=\frac{z(g * f)^{\prime}(z)}{(g * f)(z)}$. If $f \in S_{g}(h)$, then $F<h$. Now,

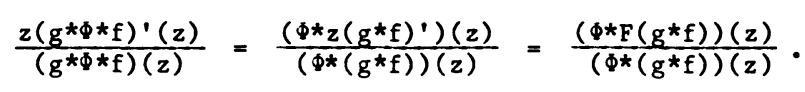

Since $f \in S_{g}(h), \quad g * f \varepsilon S^{*}(h) \subseteq S^{*}$ and it follows from Theorem 2.1 that $\frac{(\Phi * F(g * f))(z)}{(\Phi *(g * f))(z)} 1$ ies in the convex hull of $F(U)$. But $F<h$ and $h$ is convex. Therefore the convex hull of $F(U)$ is a subset of $h(U)$ and the conclusion follows.

COROLLARY 3.1: Let $\phi \varepsilon A$ be convex univalent then for every $f \varepsilon K_{g}(h), \phi \star_{f} \varepsilon K_{g}(h)$. PROOF: This follows easily from Theorem 3.2 (ii) and Theorem 3.3. 
THEOREM 3.4: $\quad \mathrm{S}_{\mathrm{g}}(\mathrm{h}) \subseteq \mathrm{S}_{\phi * \mathrm{~g}}(\mathrm{~h})$ for every convex univalent function $\phi$ with $\phi(0)=\phi^{\prime}(0)=1$.

PROOF: Let $f \varepsilon S_{g}(h)$, then by Theorem $3.3 \phi \star_{f} \varepsilon S_{g}(h)$. Hence $\frac{\left.z(g * \Phi)^{\prime}\right)^{\prime}(z)}{(g * \Phi)^{*}(z)}<h(z)$. That is $f \varepsilon S_{\Phi * g}(h)$. Normalizing condition on $\phi$ are forced because of the conditions on g's.

COROLLARY 3.2: $\mathrm{K}_{\mathrm{g}}(\mathrm{h}) \subseteq \mathrm{K}_{\Phi * \mathrm{~g}}(\mathrm{~h})$ for every convex univalent function $\phi$ in $A$.

PROOF: Follows easily from Theorem 3.2 (ii) and Theorem 3.4 .

REMARK 3.2: If $g(z)=z /(1-z)^{a+1}=k_{a+1}(z)$ and observing that $\left(h_{\gamma}{ }^{*}{ }_{a+1}\right)=K_{a}(z)$, where $h_{\gamma}(z)=\sum_{1}^{\infty} \frac{\gamma+1}{n+\gamma} z^{n}$ with $\gamma=a-1$. Then Theorem 3.4 and Corollary 3.2 reduce to the fact that $s_{a+1}(h) \subseteq s_{a}(h)$ and $K_{a+1}(h) \subseteq k_{a}(h)$ for $a>1$. These two containment results are proved respectively in [4] and [5].

It follows easily from Definition 2.3 by taking $\Psi=f$ that $\mathrm{s}_{\mathrm{g}}(\mathrm{h}) \subseteq \mathrm{C}_{\mathrm{g}}(\mathrm{h})$. We now prove that the class $C_{g}(h)$ is closed under convolution with a convex function.

THEOREM 3.5: Let $f \in C_{g}(h)$ with respect to a function $f_{1} \varepsilon S_{g}(h)$. Then for every convex univalent function $\Phi \varepsilon A, \Phi \star_{f} \varepsilon C_{g}(h)$ with respect to $\Phi \star_{f} \varepsilon S_{g}(h)$.

PROOF: It is clear from Theorem 3.3 that $\Phi *_{1} \varepsilon S_{g}(h)$. Let $F(z)=\frac{z(g * f) \cdot(z)}{(g * f)(z)}$. Since $f \varepsilon C_{g}(h)$ with respect to $f_{1} \varepsilon S_{g}(h)$, it follows that $F(z)<h(z), \forall z \varepsilon U$, in particular $\operatorname{Re} F(z)>0$, also we have $g{ }^{*} f_{1} \varepsilon S^{\star}$. Now

$$
\frac{z\left(g * \Phi *_{f}\right)^{\prime}(z)}{\left(g * \Phi f_{1}\right)(z)}=\frac{\left(\Phi *_{z}(g * f)^{\prime}\right)(z)}{\left(\Phi *\left(g * f_{1}\right)\right)(z)}=\frac{\left(\Phi * F\left(g *_{1}\right)\right)(z)}{\left(\Phi *\left(g * f_{1}\right)\right)(z)}
$$

Applying Theorem 2.1 we get Theorem 3.5 .

REMARK 3.3: If $g(z)=z /(1-z)^{a}$, and $\phi=h_{\gamma}(z)$, we get Theorem 4 of [4] as a particular case of Theorem 3.5 .

THEOREM 3.6: $\quad C_{g}(h) \subseteq C_{\Phi * g}(h)$ for every convex univalent function $\Phi \varepsilon A$.

PROOF: It follows exactly in the same way as Theorem 3.4 and is hence omitted.

REMARK 3.4: If $g(z)=z /(1-z)^{a}$ and $\Phi(z)=h_{\gamma}(z)$ with $\gamma=a-1$ we get Theorem 3 of [4].

THEOREM 3.7: (i) Let $\alpha>0$, then $\mathrm{K}_{\mathrm{g}}^{\alpha}(\mathrm{h}) \subseteq \mathrm{S}_{\mathrm{g}}(\mathrm{h})$

(ii) for $\alpha>\beta>0, K_{g}^{\alpha}(h) \subseteq K_{g}^{\beta}(h)$. 
PROOF: (i) Let $p(z)=\frac{z\left(g^{\star} f\right)^{\prime}(z)}{\left(g^{\star} f\right)(z)}$, then $J_{g}(\alpha ; f(z))=p(z)+\frac{\alpha z p^{\prime}(z)}{p(z)}$. Since $f \varepsilon K_{g}^{\alpha}(h)$ it follows that $J_{g}(\alpha ; f(z))<h(z)$. Now an application of theorem 2.2

Jives that $p(z)<h(z)$. Hence $f \in S_{g}(h)$.

(ii) The case $B=0$ is contained in (i) so assume $B>0$. Now,

$$
\begin{aligned}
J_{g}(\beta ; f(z)) & =(1-\beta) \frac{z\left(g^{\star} f\right)^{\prime}(z)}{\left(g^{\star} f\right)(z)}+\beta\left(1+\frac{z\left(g^{\star} f\right)^{\prime} \cdot(z)}{\left(g^{\star} f\right)^{\prime}(z)}\right) \\
& =\left(1-\frac{\beta}{\alpha}\right) \frac{z\left(g^{\star} f\right)^{\prime}(z)}{\left(g^{\star} f\right)(z)}+\frac{\beta}{\alpha} J_{g}(\alpha ; f(z))
\end{aligned}
$$

by (i) $\frac{z\left(g^{\star} f\right)^{\prime}(z)}{\left(g^{\star} f\right)(z)}<h(z)$ and by assumption $J_{g}(\alpha ; f(z))<h(z)$ and hence $\mathrm{J}_{\mathrm{g}}(\beta ; \mathrm{f}(z))<\mathrm{h}(\mathrm{z}) \quad$ (as $\left.\beta / \alpha<1\right)$. Therefore $\mathrm{f}_{\mathrm{g}} \mathrm{K}_{\mathrm{g}}^{\beta}(\mathrm{h})$.

REMARK 3.5: If $g(z)=z /(1-z)$ and $h(z)=\frac{1+z}{1-z}$ the first part of Theorem 3.7 reduces to the result due to Mocanu and Reade [9] that all $\alpha$-convex functions are starlike univalent and the second part of Theorem 3.7 reduces to a result of Sakaguchi [10]. If $g(z)=z /(1-z)^{a}$ then Theorem 3.7 gives Theoren 6 of Padmanabhan and Manjini [5] as a particular case.

$\begin{aligned} \text { THEOREM 3.8: } & \text { (i) } K_{g}(h) \subseteq Q_{g}(h) \subseteq C_{g}(h) \\ & \text { (ii) } f \varepsilon_{g}(h) \text { if and only if } z f^{\prime} \varepsilon C_{g}(h) .\end{aligned}$

PROOF: (1) By taking $f=\Phi$ it follows easily from the definition of the class $Q_{g}(h)$ that $k_{g}(h) \subseteq Q_{g}(h)$. To prove the other inclusion, set

$$
\begin{aligned}
& p(z)=\frac{z\left(g^{\star} f\right)^{\prime}(z)}{\left(g^{\star} \Phi\right)(z)} \text {. Then } \\
& p(z)+\frac{z p^{\prime}(z)}{\frac{z\left(g^{\star} \Phi\right)^{\prime}(z)}{\left(g^{\star} \Phi\right)(z)}}=\frac{\left[z\left(g^{\star} f\right)^{\prime}(z)\right]^{\prime}}{\left(g^{\star} \Phi\right)^{\prime}(z)}
\end{aligned}
$$

If $f \varepsilon_{g}(h)$ then there exists a $\Phi \varepsilon K_{g}(h)$ such that the right hand side of $(3.3)$ and hence the left hand side of (3.3) is subordinate to $h(z)$.

Since $\Phi \varepsilon \mathrm{K}_{\mathrm{g}}(\mathrm{h}) \subseteq \mathrm{S}_{\mathrm{g}}(\mathrm{h})$ we have $\operatorname{Re} \frac{z(\mathrm{~g} \star \Phi))^{\prime}(z)}{\left(\mathrm{g}^{\star \Phi}\right)(z)}>0$ in $\mathrm{J}$. Hence applying Theorem 2.3 we get $p(z)<h(z)$. That is $f \in C_{g}(h)$.

To prove (ii) we have for any two functions $f$ and $\Phi$ satisfying the non-zero convolution conditions that

$$
\frac{z\left[z\left(g^{\star} f\right)^{\prime}(z)\right]^{\prime}}{z\left(g^{\star} \Phi\right)^{\prime}(z)}=\frac{z\left(g^{\star} z f^{\prime}\right)^{\prime}(z)}{\left(g^{\star} z^{\prime} \Phi^{\prime}\right)(z)}
$$


Now, if $f \in Q_{g}(h)$ with respect to a function $\Phi \varepsilon K_{g}(h)$, then the left hand side of 3.4 is subordinate to $h(z)$. Now $z \Phi^{\prime} \varepsilon S_{g}(h)$ by Theorem 3.2 (ii) and hence by the definition of $C_{g}(h)$ and by (3.4) $z f^{\prime} \varepsilon C_{g}(h)$. Conversely, if $z f^{\prime} \varepsilon C_{g}(h)$, then there exists a function $\Phi_{1} E S_{g}(h)$ such that

$$
\frac{z\left(g * z f^{\prime}\right)^{\prime}(z)}{\left(g * \Phi_{1}\right)(z)}<h(z) .
$$

Now there exists a $\Phi \varepsilon K_{g}(h)$ such that $z^{\prime}=\Phi_{1}$ and hence the RHS of 3.4 is subordinate to $h(z)$ which implies the LHS of 3.4 is subordinate to $h(z)$ which in turn gives that $f \in Q_{g}(h)$.

REMARK 3.6: When $g(z)=z / 1-z$ Theorem 3.8 reduces to Theorem 1 of Noor and Thomas [8]. By Theorems 3.2 (i) and 3.8 and from the observation we made just before Theorem 3. 5 we get the following inclusions

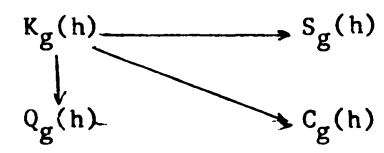

where, the direction of the 'arrows' indicate the respective inclusions.

THEOREM 3.9: If $f \varepsilon_{\mathrm{g}}(\mathrm{h})$, then for every convex univalent function $\Phi \varepsilon A, \Phi * f \varepsilon_{\mathrm{g}}(\mathrm{h})$.

THEOREM 3.10: $Q_{g}(h) \subseteq Q_{\Phi *_{g}}(h)$ for every convex univalent function $\Phi \varepsilon A$, in particular $Q_{a+1}(h) \subseteq Q_{a}(h)$ for $a>1$.

PROOF: The proofs of the above Theorems 3.9 and 3.10 are omitted because it will follow from 3.8 and the corresponding theorems for the class $\mathrm{C}_{\mathrm{g}}(\mathrm{h})$.

4. CONCLUDING REMARKS.

It would be interesting to find a necessary and sufficient condition on the function $g(z)$ so that $f * g$ univalent implies $f$ is univalent.

\section{REFERENCES}

1. GoODMAN, A.W., Univalent Functions, Vol. I and II, Mariner Publishing Co., Inc., Florida, Tampa, USA (1984).

2. BARNARD, R.W. and KELLOGG, C., Applications of Convolution Operators to Problems in Univalent Function Theory, Michigan, Math. J. 27 (1980), 81-94.

3. EenigenbuRG, P., MIller, S.S., MOCANU, P.T. and READE, M.0.. On a Briet-Boquet Differential Subordination, Revue. Roum. Pure et Applique Vo1. 29 (1984), No. 9, 1-7.

4. PADMANABHAN, K.S. and PARVATHAM, R., Some Applications of Differential Subordination, Bul1. Austral. Math. Soc., Vol. 32, No. 3 (1985), 321-330.

5. PADMANABHAN, K.S. and MANJINI, R., Certain Applications of Differential Subordination, Pubi de L'Institut Mathe' Matique, Vol. 39 (53) (1986) 107118. 
6. OWA, S., FUKIU, S., SAKAGUCHI, K. and OGAWA, S., An Application of the Ruschewegh Derivatives, Internat. J. Math. and Math. Sc1., Vol.9, No. 4, (1986), 721730.

7. RUSCHEWEGH, ST.. Linear Operators Between Classes of Pre-Starlike Functions, Comment. Math. Helvetici, Vol. 52 (1977), 497-509.

8. INAYAT NOOR, K. and THOMAS, D.K., On Quasi-Convex Univalent Functions, Internat. J. Math. and Math. Sci., Vol. 3 (1980), 255-266.

9. MOCANU, P.T. and READE, M.0., On Generalized Convexity in Conformal Mappings, Revue Roum. Math. Pure et Appl, Vo1. 16 (1971), 1541-1544.

10. SAKAGUCHI, K.S., A Note on P-Valent Functions, J. Math. Soc. Japan, Vol. 14 (1962), 312-321. 


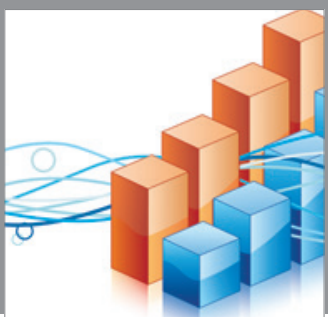

Advances in

Operations Research

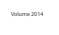

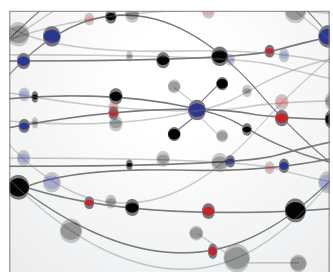

\section{The Scientific} World Journal
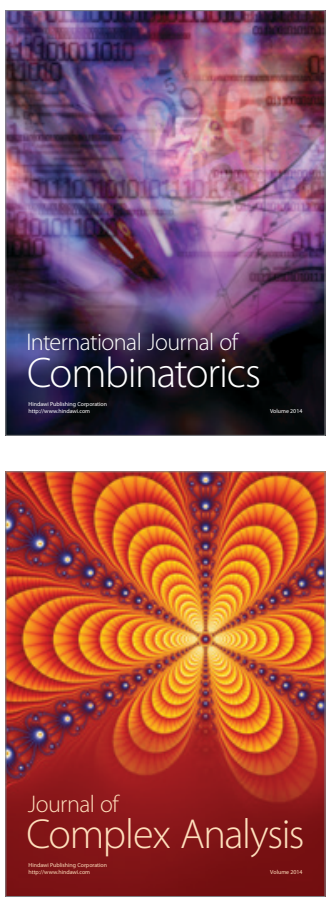

International Journal of

Mathematics and

Mathematical

Sciences
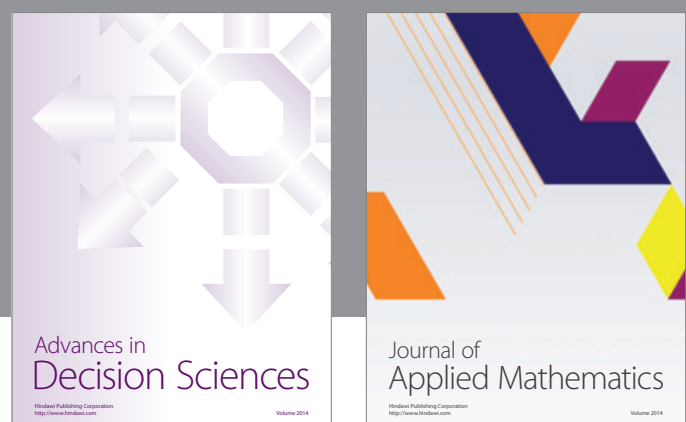

Journal of

Applied Mathematics
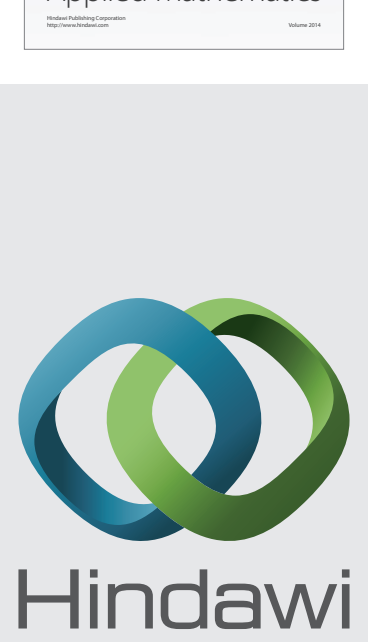

Submit your manuscripts at http://www.hindawi.com
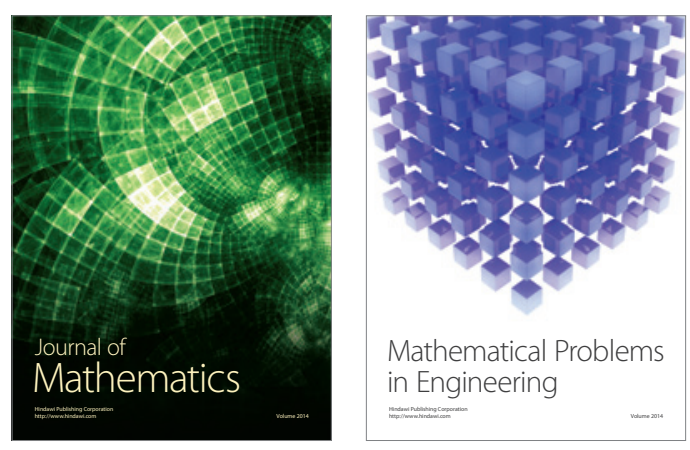

Mathematical Problems in Engineering
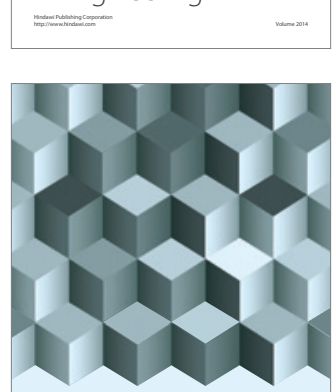

Journal of

Function Spaces
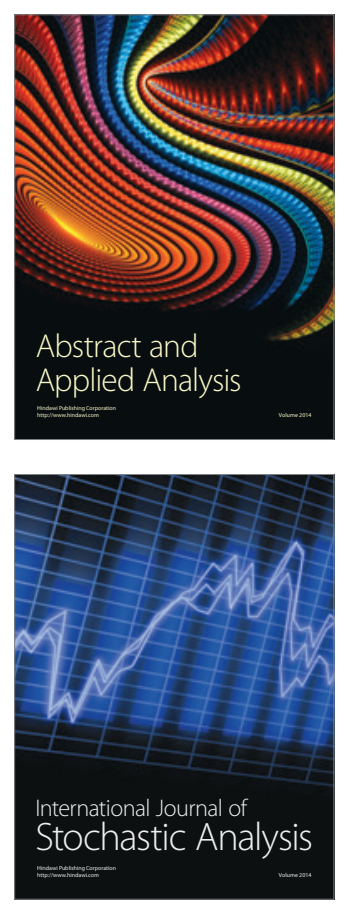

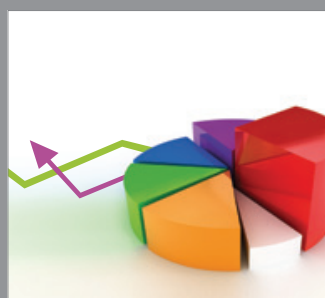

ournal of

Probability and Statistics

Promensencen
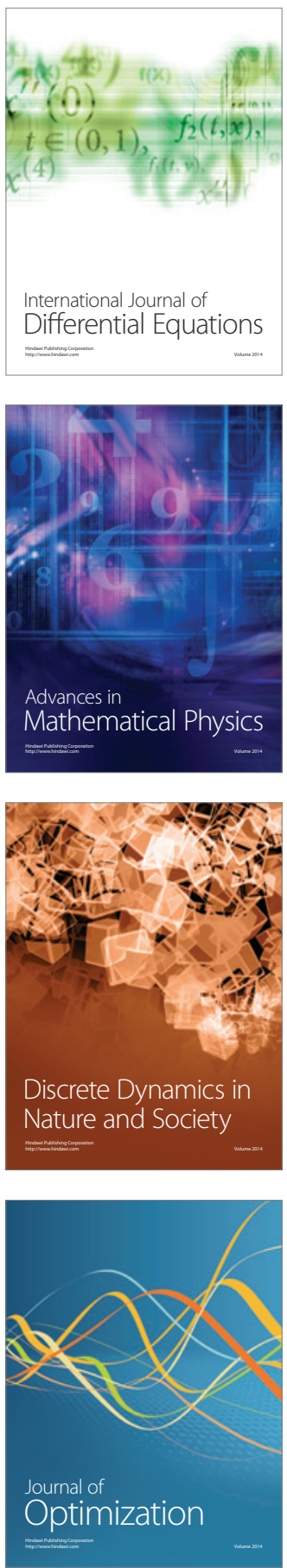